\title{
Patient and physician preferences for attributes of coronary revascularization
}

This article was published in the following Dove Press journal:

Patient Preference and Adherence

\section{Carlos Alberto da \\ Silva Magliano' \\ Andrea Liborio Monteiro ${ }^{2}$ \\ Bernardo Rangel Tura' \\ Claudia Silvia Rocha \\ Oliveira' \\ Amanda Rebeca de Oliveira \\ Rebelo' \\ Claudia Cristina de \\ Aguiar Pereira $^{3}$}

'HTA Department, National Institute of Cardiology, Rio de Janeiro, Brazil;

${ }^{2}$ Department of Pharmacy Systems,

Outcomes and Policy, College of

Pharmacy, University of Illinois at

Chicago, Chicago, IL, USA; ${ }^{3}$ The

National School of Public Health

Sergio Arouca ENSP/Fiocruz,

Rio de Janeiro, Brazil
Correspondence: Carlos Alberto da Silva Magliano

Rua das Laranjeiras 374, 5 andar, HTA Department, National Institue of Cardiology, Rio de Janeiro, Brazil, CEP 22240-006

Tel +55 2I 996802076

Fax +55 21 25379739

Email carlosincnats@gmail.com
Background: Patients with a diagnosis of coronary artery disease (CAD) may face important decisions regarding treatment options, with the "right choice" depending on the relative weights of risks and benefits. Studies performed as discrete choice experiments are used to estimate these weights, and attribute selection is an essential step in the design of these studies. Attributes not included in the design cannot be analyzed. In this study, we aimed to elicit, rank, and rate attributes that may be considered important to patients and physicians who must choose between angioplasty and surgery for coronary revascularization.

Methods: The elicitation process involved performing a systematic review to search for attributes cited in declared preference studies in addition to face-to-face interviews with cardiologists and experts. The interviews were audio-recorded in digital format, and the collected data were transcribed and searched to identify new attributes. The criterion used to finish the data collection process was sampling saturation.

Results: A systematic review resulted in the selection of the following 14 attributes: atrial fibrillation, heart failure, incision scar, length of stay, long-term survival, myocardial infarction, periprocedural death, postoperative infection, postprocedural angina, pseudoaneurysm, renal failure, repeat coronary artery bypass grafting, repeat percutaneous coronary intervention, and stroke. The interviews added no new attributes. After rating, we identified significant differences in the values that patients and cardiologists placed on renal insufficiency $(p<0.001)$, periprocedural death $(p<0.001)$, and long-term survival $(p<0.001)$.

Conclusion: Decisions regarding the best treatment option for patients with CAD should be made based on differences in risk and the patient's preference regarding the most relevant endpoints. We elicited, ranked, and rated 14 attributes related to CAD treatment options. This list of attributes may help researchers who seek to perform future preference studies of CAD treatment options.

Keywords: preference, ranking, rating, coronary, angina

\section{Introduction}

Coronary artery disease (CAD) is the leading cause of mortality worldwide. For patients with symptomatic CAD refractory to medical therapy, two treatment options are available: percutaneous coronary intervention (PCI) and coronary artery bypass grafting (CABG). Each of these therapies has a different risk and benefit profile. Approximately $12 \%$ of affected patients are eligible for either PCI or CABG. ${ }^{1}$ Knowing the preferences of the patients who face this scenario may be relevant to decisionmaking because the available treatment options are different, and the stakeholders may value them unequally.

Determining patient preferences may provide insights that lead to more effective management strategies since which treatment is "right" depends on the relative weight 
the patient gives to the risks and benefits of the different treatment strategies. In this study, we aimed to elicit, rank, and rate attributes that may be considered important to patients and physicians who face choosing between PCI and CABG.

This paper describes a qualitative study that was designed to identify which attributes are important to patients and cardiologists. These attributes may help future studies aimed at exploring the preference for coronary revascularization, such as discrete choice experiments (DCEs). DCEs are increasingly used as a method to determine patient preferences, and attribute selection is an important step that is rarely reported in detail. ${ }^{2}$

\section{Methods}

The first step in this study was to elicit attributes. The second step was to rank and rate the attributes selected by patients and physicians. Finally, we analyzed the attributes to identify differences between these stakeholders' perspectives.

\section{Attribute elicitation}

The elicitation process involved performing a systematic review in addition to conducting face-to-face interviews with patients and experts.

\section{Systematic review}

We performed a systematic review of published studies related to declared preferences for PCI or CABG in patients with a diagnosis of CAD. The MEDLINE, Embase, and LILACS databases were searched for studies published on or before 10 August 2017. Our search terms consisted of keywords for coronary revascularization as well as methods used to assess patient preferences (eg, "patient reported outcome," "patient satisfaction," "best-worst scaling," "Maxdiff," "discrete choice experiment," "preference," "conjoint studies," "conjoint choice experiment," "vignette," “choice," "willingness-to-pay," and "conjoint analysis").

\section{Patient interviews to elicit attributes}

Data collection was conducted by trained interviewers, including one cardiologist and two nurses, at the National Institute of Cardiology, Rio de Janeiro, Brazil. These professionals had no previous contact with the patients, and none of them was responsible for the care of the included patients.

In this study, we used a convenience sample of hospitalized patients with stable CAD, most of whom were waiting for a coronary revascularization procedure. Respondents were selected randomly based on their ward number using a list of random numbers that were generated in Microsoft Excel. We chose patients with CAD since we assumed that they would be more committed to the interviews than any alternative sample population.

Eligibility to participate in the study was defined as follows: a diagnosis of CAD, willingness to participate in a face-to-face interview during which the patient would answer questions about their preferences regarding possible treatment complications, provided written informed consent, and currently being considered for a revascularization treatment. Participants were deemed ineligible if they had already undergone surgery or angioplasty or if they viewed themselves as unable to understand the experiment. Ethical approval was obtained from the National Institute of Cardiology Ethical Department (Comitê de ética e pesquisa do Instituto Naional de Cardiologia), and written informed consent was obtained from each study participant (CAAE number 63684017.0.0000.5240).

One-to-one interviews were conducted between August 1 and November 20,2017. Patients were asked to imagine that they were sharing in the decision-making process regarding which treatment option was better for them between PCI and CABG. They were then asked to answer a semi-structured questionnaire. Open-ended questions were presented in the following sequence: 1) Do you know the reason for your hospitalization? 2) What benefits do you expect to achieve from the treatment? 3) Which risks or complications could occur with the treatment? 4) What most concerns you regarding the treatment? 5) Considering that there is no difference in mortality between the two, which procedure would you prefer? 6) What are the differences between surgery and angioplasty?

The latter part of the questionnaire included questions regarding demographic information (age, gender, ethnicity, income, and level of education).

\section{Cardiologist interviews to elicit attributes}

All interviews were completed by the first author (CM), who is a cardiologist. The study population was a convenient sample of cardiologists with expertise in CAD.

Physician eligibility was determined according to the following criteria: specialist in cardiology, willing to participate in a face-to-face interview and answer questions about preferences regarding possible treatment complications, and willing to provide informed consent.

The experts were asked to enumerate the risks and benefits they usually consider when exploring the trade-offs between PCI and CABG. 


\section{Qualitative analysis of interviews}

The interviews were audio-recorded in digital format, and the data collected were qualitatively analyzed. First, the data were transcribed integrally and properly contrasted with the audio versions to detect errors. Then, we reviewed the data to identify any attribute that had not been previously identified in the systematic review. We then sought to identify the terms that were most commonly used by patients to describe clinical outcomes. The criterion used to finish data collection was sampling saturation. ${ }^{3}$

\section{Ranking}

The attributes selected in the previous step were randomly and individually presented to patients and physicians. We used cards that were similar in color and size, with each card containing the name of an attribute in layperson's terms. The amount of explanatory/background information was equivalent for all attributes, and this information was presented according to a pre-established script to avoid overstating the importance of any single attribute.

The following pre-established question was presented to all patients:

Consider you have angina, a chest pain that indicates a heart problem. Your treatment options are medicine, angioplasty (catheterization with stent placement) or bypass surgery. These different treatments are related to different benefits and risks. I have a list of possible complications resulting from these treatments, and I would like to know how concerned you are in relation to each one. Please, put in order of relevance from one to fourteen your concerns, ie, one being the most concerned to fourteen the least concerned.

The following similar pre-established question was presented to all physicians:

Imagine that you are responsible for defining the treatment of a patient who is complaining about angina. The available treatment options include adjusting medications, angioplasty with stents or coronary bypass surgery. These different treatments are related to different benefits and risks. I have a list of possible complications from these treatments and I would like to know how concerned you are about each one. Please, put in order of relevance from one to fourteen your concerns, ie, one being the most concerned to fourteen the least concerned.

\section{Rating}

After ranking, each participant received 10 identical black dots. They were instructed to distribute the dots in such a manner that the most relevant attributes received proportionally more points. The participant was asked to define the proportion by considering the relative importance of each attribute. There was no limit to how many points each attribute could receive. It was possible to reorder the attributes during this phase. This method is known as dotmocracy (Figure 1).

\section{Statistics}

Descriptive statistics were used to summarize the sociodemographic and clinical characteristics of the sample. We summarized the data as means, medians, or percentages, as indicated. An unpaired $t$-test was used for comparisons between group data. All procedures were performed in accordance with the ethical standards of the responsible committee on human experimentation (institutional and national) and with the Helsinki Declaration of 1964, as revised in 2013. Informed consent was obtained from all patients prior to their inclusion in the study.

\section{Results}

The interviews were $\sim 15 \mathrm{~min}$ in duration. We enrolled 76 respondents, comprising 22 cardiologists and 54 patients (32 for elicitation and 22 for ranking and rating). Overall, the majority of respondents were male, Caucasian, retired, married, and religious (Table 1). All physicians were cardiologists and had a mean \pm SD $15.6 \pm 1.2$ years of clinical experience.

\section{Attribute selection in the systematic review}

We used a systematic review to search for stated preference studies that evaluated CAD treatments. Both patient and physician preferences were surveyed. Of a total of 735 citations (436 from MEDLINE and 299 from Embase), 44 articles were eligible for full-text review. Six studies ${ }^{1,4-8}$ that met our inclusion criteria were included in the final report (Figure 2). The attributes most often cited in these studies coincided with those already used in clinical trials: death, ${ }^{4,6-8}$ stroke, ${ }^{4,6-8}$ myocardial infarction, ${ }^{4,7}$ and repeat revascularization. ${ }^{1,6-8}$ Other attributes examined within these studies included heart attack, ${ }^{6}$ hospitalization, ${ }^{6}$ repeat PCI, ${ }^{4}$ repeat $\mathrm{CABG},{ }^{4}$ atrial fibrillation, ${ }^{4}$ mediastinitis, ${ }^{4}$ postoperative chronic pain,,${ }^{1,4}$ postprocedural angina, ${ }^{4,6}$ length of stay, ${ }^{1,4}$ duration of recovery,,${ }^{1,4}$ depression, ${ }^{4}$ procedural cost,${ }^{4}$ incision scar, ${ }^{1,4,5}$ pseudoaneurysm, ${ }^{4}$ pneumonia, ${ }^{4}$ long-term use of clopidogrel, ${ }^{4}$ renal failure, ${ }^{4}$ acute respiratory distress syndrome,${ }^{4}$ bleeding, ${ }^{4}$ symptom control, medication side effects, comfort in taking medication, well-being, the ability to do things, the prolonging of life, and cure. ${ }^{5}$ 


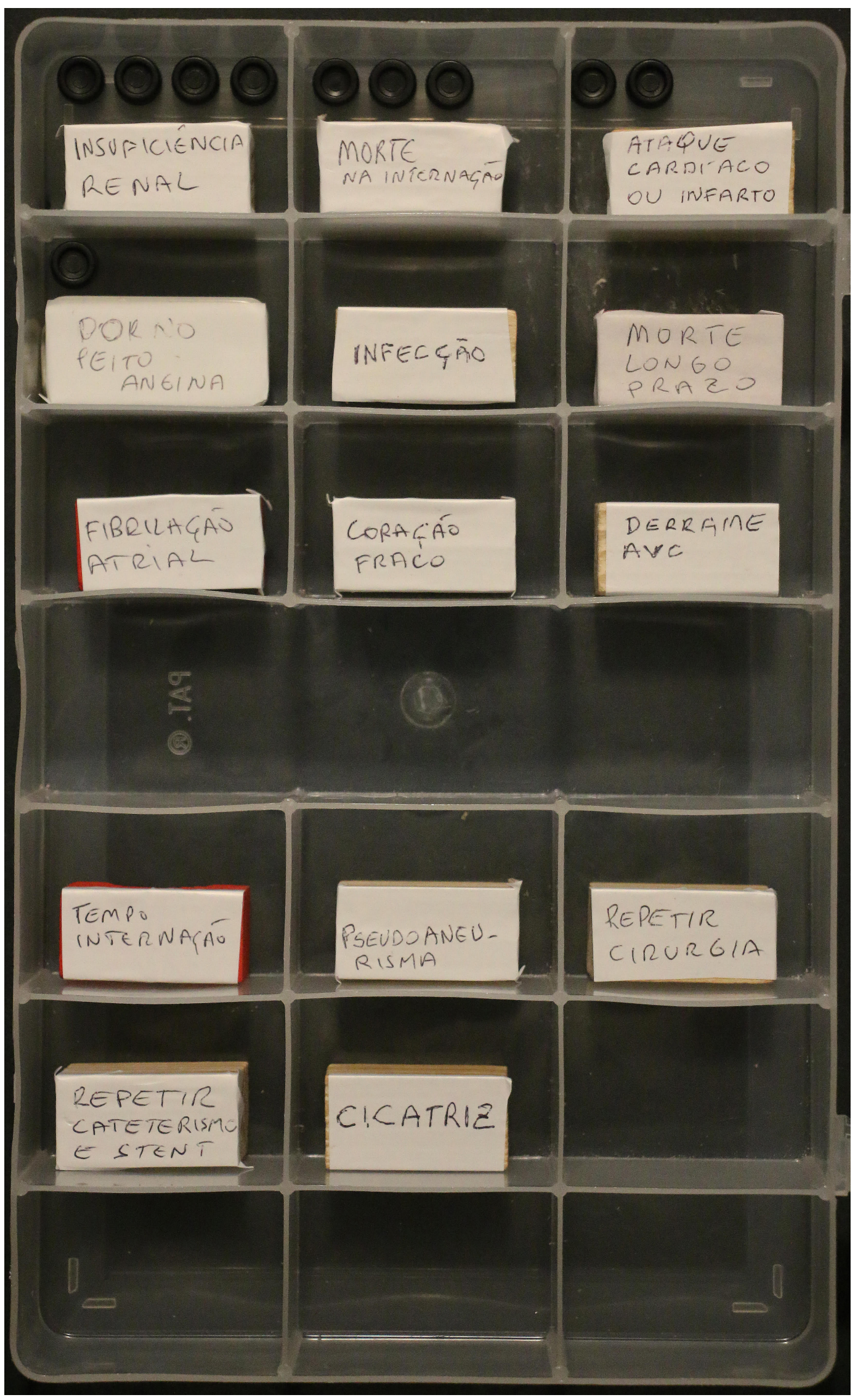

Figure I The dotmocracy rating method. 
Table I Patients' sociodemographic characteristics

\begin{tabular}{ll}
\hline Characteristic & N (\%) \\
\hline Number of participants & 54 \\
Gender & \\
Male & $40(74)$ \\
Female & $14(26)$ \\
Age (years), mean \pm SD & $62.5 \pm 8.6$ \\
Religion & \\
Catholic & $25(46)$ \\
Evangelical & $16(30)$ \\
Spiritism & $6(11)$ \\
Other & $7(13)$ \\
Ethnicity & \\
White & $27(50)$ \\
Pardo & $22(41)$ \\
Black & $5(9)$ \\
Years of education & \\
I-5 & $13(24)$ \\
$6-10$ & $12(22)$ \\
II-I3 & $18(33)$ \\
$>$ I3 & $11(21)$ \\
Retired & $37(69)$ \\
Married & $37(69)$ \\
Monthly income (R\$), median $\pm S D$ & $2205.37 \pm 2890.26$ \\
\hline Not
\end{tabular}

Note: Data are shown as $\mathrm{n}(\%)$ unless otherwise indicated.

Procedural and other costs, such as medications, were avoided as possible attributes in this study because the included patients were being treated in the Brazilian public health system and therefore had no copayments. Prolonged use of medications or their side effects were also not included as attributes because our goal was to study preferences between angioplasty and surgery.

We selected the following 14 attributes based on the attributes identified in the systematic review: 1) atrial fibrillation; 2) heart failure; 3) incision scar; 4) length of stay; 5) long-term survival; 6) myocardial infarction; 7) periprocedural death;

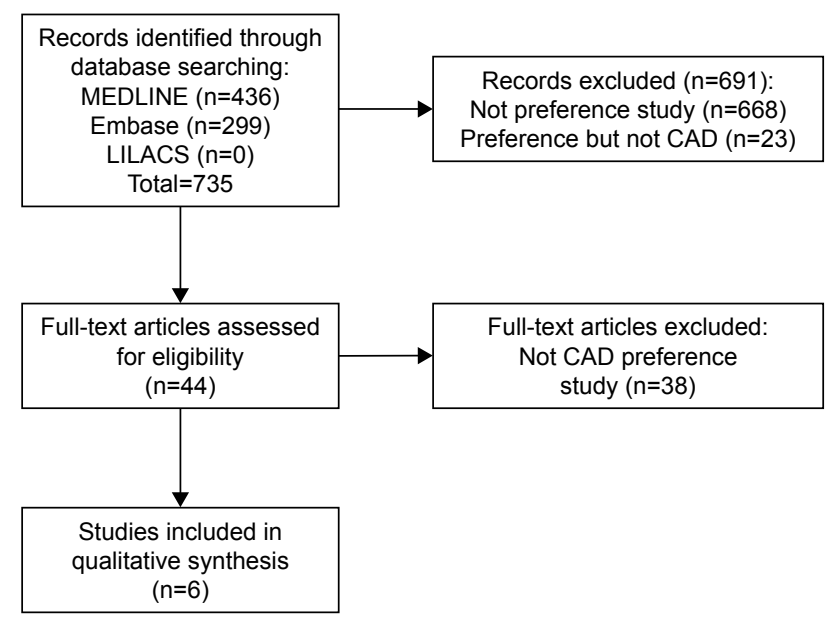

Figure 2 Flowchart of the systematic review. Abbreviation: CAD, coronary artery disease.
8) postoperative infection; 9) postprocedural angina; 10) pseudoaneurysm; 11) renal failure; 12) repeat $\mathrm{CABG}$; 13) repeat PCI; and 14) stroke (Table 2).

\section{Attributes selected from patient and physician interviews}

Interviews with cardiologists and patients did not add new attributes. Notably, the patients exhibited discomfort when encouraged to cite possible complications and claimed that they had not spoken to their physicians about these. The complications most prevalent from a patient perspective were death and stroke, which were cited by $68.75 \%$ and $50 \%$ of the patients, respectively.

\section{Attribute importance}

After ranking and rating the identified attributes, our survey revealed that in some attributes, there was a major discordance between patients and cardiologists. Periprocedural death was ranked as the most important outcome by cardiologists, while renal failure was the most important to patients. Incision scars were considered the least important for both groups. There was a significant difference between patients and cardiologists in the value they placed on renal insufficiency $(p<0.001)$, periprocedural death $(p<0.001)$, and long-term survival $(p<0.001)$. The results are shown in Table 3.

\section{Discussion}

We would like to highlight some of the important findings of this study. First, neither the patients nor the physicians considered all clinical endpoints equal, and future trials should therefore be careful when considering composite endpoints. Second, patients and physicians do not weigh many relevant cardiovascular outcomes similarly (eg, renal failure, periprocedural death, long-term survival, and atrial fibrillation), and this should be considered during decisionmaking. Third, repeat revascularization is not highly valued by either doctors or patients and should therefore not substantially impact the decision-making process.

In this study, we identified some important aspects that should be explored in future trials. The attributes that were more frequently cited in the systematic review of preference studies coincided with the attributes already used in clinical trials. The patient and physician interviews did not add any new attributes. This finding may indicate that these attributes are indeed the most relevant, or it may reflect the impact of previously published trial outcomes on current medical reasoning. One relevant question is that of whether 
Table 2 Definitions of attributes

\begin{tabular}{|c|c|}
\hline Attribute & Definition \\
\hline Atrial fibrillation & Permanent atrial fibrillation, patient received anticoagulation \\
\hline Heart failure* & $\begin{array}{l}\text { Symptomatic heart failure, NYHA class III or IV. Class III: marked limitation of physical activity in which less } \\
\text { than ordinary activity results in fatigue, palpitation, dyspnea, or angina pain, but the person is comfortable at } \\
\text { rest. Class IV: inability to carry on any physical activity without discomfort, symptoms of heart failure or angina } \\
\text { syndrome even when at rest, and increased discomfort if any physical activity is undertaken }\end{array}$ \\
\hline Incision scar & Median sternotomy incision scar \\
\hline Length of stay & Hospitalization length of stay \\
\hline Long-term survival & 5-year survival after treatment \\
\hline Myocardial infarction & $\begin{array}{l}\text { Myocardial infarction not related to death. "Commonly known as a heart attack, occurs when blood flow } \\
\text { decreases or stops to a part of the heart. The symptoms include chest pain or discomfort, shortness of breath, } \\
\text { nausea, feeling faint, a cold sweat, or feeling tired." } 10\end{array}$ \\
\hline Periprocedural death & Death within 30 days after treatment \\
\hline Postoperative infection & Infection after surgery, including skin infections, pneumonia, or mediastinitis \\
\hline Postprocedural angina & $\begin{array}{l}\text { Return of angina symptoms despite treatment, including chest pain or pressure, which is usually related to } \\
\text { exercise or emotional stress }\end{array}$ \\
\hline Pseudoaneurysm & $\begin{array}{l}\text { Femoral pseudoaneurysm. A pseudoaneurysm, also known as a false aneurysm, is a collection of blood that forms } \\
\text { between the two outer layers of an artery. It is caused by an injury to the femoral vessel that occurs during } \\
\text { angioplasty. Correction may require surgery }\end{array}$ \\
\hline Renal failure & $\begin{array}{l}\text { Chronic kidney failure, also known as renal failure or renal insufficiency, is a medical condition in which the } \\
\text { kidneys do not work properly, and dialysis is needed to perform their function }\end{array}$ \\
\hline Repeat CABG & The necessity to redo surgery \\
\hline Repeat $\mathrm{PCl}$ & The necessity to redo angioplasty \\
\hline Stroke & $\begin{array}{l}\text { Permanent stroke. Signs and symptoms of a stroke may include an inability to move, the loss of feeling on one side } \\
\text { of the body, problems understanding or speaking, feeling like the world is spinning, or loss of vision to one side }\end{array}$ \\
\hline
\end{tabular}

Note: *https://emedicine.medscape.com/article/2500037-overview."

Abbreviations: NYHA, New York Heart Association; CABG, coronary artery bypass graft; PCl, percutaneous coronary intervention.

physicians make their decisions based exclusively on the outcomes used in previous trials rather than considering all possible patient-relevant outcomes.

Another important issue related to outcomes is the use of composite endpoints. Death, stroke, myocardial infarction, and repeat revascularization are frequently grouped into a

Table 3 Ranking and rating of attributes according to patients and cardiologists

\begin{tabular}{|c|c|c|c|c|c|}
\hline \multirow[t]{2}{*}{ Attribute } & \multicolumn{2}{|l|}{ Patients } & \multicolumn{2}{|c|}{ Cardiologists } & \multirow[t]{2}{*}{ p-value } \\
\hline & Ranking & $\overline{\text { Rating }}$ & Ranking & $\overline{\text { Rating }}$ & \\
\hline Renal failure & Ist & 50 & 6th & 10 & $<0.001$ \\
\hline Periprocedural death & 2nd & 43 & Ist & 72 & $<0.001$ \\
\hline Stroke & $3 r d$ & 25 & $3 r d$ & 26 & NS \\
\hline Repeat surgery & 4th & 19 & 8th & 8 & NS \\
\hline Myocardial infarction & 5 th & 16 & 4th & 18 & NS \\
\hline Heart failure & 6th & 15 & 5 th & 15 & NS \\
\hline Angina & 7th & 12 & 9th & 4 & NS \\
\hline Atrial fibrillation & 8th & 10 & 12 th & I & 0.01 \\
\hline Postoperative & 9th & 9 & 7th & 4 & NS \\
\hline \multicolumn{6}{|l|}{ infection } \\
\hline Long-term survival & 10th & 9 & 2nd & 53 & $<0.001$ \\
\hline Length of stay & II th & 4 & IIth & 2 & NS \\
\hline Pseudoaneurysm & 12 th & 2 & 13th & I & NS \\
\hline Repeat angioplasty & 13th & 2 & 10th & 2 & NS \\
\hline Incision scar & 14th & 0 & 14 th & 0 & NS \\
\hline
\end{tabular}

Abbreviation: NS, not significant. category called major adverse cardiovascular events (MACE). The use of MACE has been justified as an attempt to capture the overall treatment effect. The main advantage of using composite outcomes is that it reduces the duration, sample size, and costs of clinical trials. ${ }^{1,6}$ In this study, we found that these outcomes are weighted differently, and this represents one limitation of the use of MACE as a composite endpoint. This finding is in accordance with those presented in the literature. ${ }^{4,6,7}$

It is important to note that even though the difference was not statistically significant ( $p=0.054$ ), the patients rated "repeat surgery" as the fourth most important attribute, and they gave it more than twice the points given by physicians, who considered it the eighth most important. In addition, one endpoint that is very commonly used in composite analysis, "repeat PCI," was viewed as one of the least important by both patients (13 out of 14) and physicians (10 out of 14). This finding is similar to that of Pandit et al. ${ }^{4}$ Based on these findings, future studies should avoid grouping "repeat surgery" and "repeat PCI" in addition to avoiding the use of the combined outcomes of death, stroke, myocardial infarction, and "repeat revascularization" because these outcomes are valued differently by patients and physicians.

We considered it essential to clarify the clinical meaning of the endpoints provided during the interviews. For example, 
stroke was presented as permanent stroke. This is not likely to be the reality in many clinical trials in which there are several different types of stroke, which can range from a mild transient ischemic event to a large disabling neurological insult. Similarly, myocardial infarction may range from a trivial troponin rise to severe ventricular dysfunction. Future studies should report results stratified by attributes and clinical relevance.

Stroke was identified as an important attribute and was the third most important to both patients and physicians. However, it was not more important than death. This finding is different from that reported in the Pandit and Ahmad studies. ${ }^{4,7}$ Future trials should explore cultural aspects that are potentially related to differences in preferences between populations.

Patients and physicians weighted some attributes differently. Patients appear to place considerably less value on "long-term survival." The Kipp study ${ }^{8}$ reached a similar conclusion, wherein patients chose PCI over CABG across nearly all hypothetical PCI risk scenarios, while physicians were significantly less likely to choose PCI over CABG. During the decision-making process, physicians must recognize that there are possible divergences in the weighted preferences for some relevant attributes, and they should therefore seek the patient's opinion during this process.

Our study does have several limitations. The number of attributes selected could have resulted in respondent fatigue. Furthermore, the outcome definitions and survey structure were tested in pilot studies but were not validated. Our study was not powered for a subgroups analysis. The patients selected were from a convenience sample from a public health hospital in Brazil, and most of the participants were at the lower end of educational completion, which limits the ability to generalize the study's conclusions to the overall population. On the other hand, these findings highlight the necessity of elicitation preferences in each scenario before making recommendations.

Another important limitation of our attribute ranking analysis was the absence of attribute levels. This was because we believed that presenting several attributes with different levels would result in excessive cognitive demand. Thus, the attributes were provided with no information regarding risk rates, and we must acknowledge that this could have impacted the results.

As cited by Ciccone et al, building on treatment recommendations should take a broad perspective and consider the medical, social, behavioral, and emotional impact on the patient's quality of life. ${ }^{9}$ The current guidelines and recommendations regarding $\mathrm{CAD}$ treatment seem to be based on endpoints that do not properly represent patient preferences. Further developing what is known about patients' preferences, values, and fears may better inform policy and enable physicians to be more accountable to the public.

\section{Conclusion}

It is vital that decision makers understand how patients value the aspects associated with a health-care intervention. Incorporating their values may ultimately result in clinical and policy decisions that better reflect patient preferences.

In this study, we elicited, ranked, and rated 14 attributes related to $\mathrm{CAD}$ treatment options. Patients and cardiologists valued many of these attributes differently. This list of attributes may help researchers seeking to use discrete choice experiments to develop future preference studies related to CAD treatment options.

\section{Disclosure}

The authors report no conflicts of interest in this work.

\section{References}

1. Hornberger J, Bloch DA, Hlatky MA, Baumgartner W. Patient preferences in coronary revascularization. Am Heart J. 1999;137(6):1153-1162.

2. Bridges JF, Hauber AB, Marshall D, et al. Conjoint analysis applications in health - a checklist: a report of the ISPOR Good Research Practices for Conjoint Analysis Task Force. Value Health. 2011;14(4):403-413.

3. Prakke H, Wurster J. [Quality criteria for qualitative research]. Pflege. 1999;12(3):183-186.

4. Pandit J, Gupta V, Boyer N, Yeghiazarians Y, Ports TA, Boyle AJ. Patient and physician perspectives on outcomes weighting in revascularization. The POWR study. Int J Cardiol. 2014;177(2):513-514.

5. Bowling A, Culliford L, Smith D, Rowe G, Reeves BC. What do patients really want? Patients' preferences for treatment for angina. Health Expect. 2008;11(2):137-147.

6. Chow RD, Wankhedkar KP, Mete M. Patients' preferences for selection of endpoints in cardiovascular clinical trials. J Community Hosp Intern Med Perspect. 2014;4.

7. Ahmad Y, Nijjer S, Cook CM, et al. A new method of applying randomised control study data to the individual patient: a novel quantitative patient-centred approach to interpreting composite end points. Int J Cardiol. 2015;195:216-224.

8. Kipp R, Lehman J, Israel J, Edwards N, Becker T, Raval AN. Patient preferences for coronary artery bypass graft surgery or percutaneous intervention in multivessel coronary artery disease. Catheter Cardiovasc Interv. 2013;82(2):212-218.

9. Ciccone MM, Aquilino A, Cortese F, et al. Feasibility and effectiveness of a disease and care management model in the primary health care system for patients with heart failure and diabetes (Project Leonardo). Vasc Health Risk Manag. 2010;6:297-305.

10. Ibanez B, James S, Agewall S, et al. ESC Guidelines for the management of acute myocardial infarction in patients presenting with ST-segment elevation. Revista espanola de cardiologia. 2017;70(12): 1082.

11. Ho KK, Pinsky JL, Kannel WB, Levy D. The epidemiology of of heart failure: the Framingham study. J Am Coll Cardiol. 1993;(4 suppl A): $6 \mathrm{~A}-13 \mathrm{~A}$. 


\section{Publish your work in this journal}

Patient Preference and Adherence is an international, peer-reviewed, open access journal that focuses on the growing importance of patient preference and adherence throughout the therapeutic continuum. Patient satisfaction, acceptability, quality of life, compliance, persistence and their role in developing new therapeutic modalities and compounds to optimize

clinical outcomes for existing disease states are major areas of interest for the journal. This journal has been accepted for indexing on PubMed Central. The manuscript management system is completely online and includes a very quick and fair peer-review system, which is all easy to use. Visit http://www. dovepress.com/testimonials.php to read real quotes from published authors.

Submit your manuscript here: http://www.dovepress.com/patient-preference-and-adherence-journal 